Published in final edited form as:

Surg Oncol Clin N Am. 2017 April ; 26(2): 293-312. doi:10.1016/j.soc.2016.10.002.

\title{
Novel Targeted Therapies for Esophagogastric Cancer
}

\author{
Steven B. Maron, M.D. [Fellow] and \\ University of Chicago Comprehensive Cancer Center, Section of Hematology/Oncology, $5841 \mathrm{~S}$. \\ Maryland Avenue, Chicago, IL 60637 \\ Daniel V.T. Catenacci, M.D. [Associate Director, Gastrointestinal Oncology Program, \\ Assistant Professor of Medicine] \\ University of Chicago Comprehensive Cancer Center, Section of Hematology/Oncology, $5841 \mathrm{~S}$. \\ Maryland Avenue, Chicago, IL 60637
}

\section{Synopsis}

Gastroesophageal cancer (GEC) remains a major cause of cancer-related mortality worldwide.

Although the incidence of distal gastric adenocarcinoma (GC) is declining in the United States, proximal esophagogastric junction adenocarcinoma (EGJ) is rising in incidence. GEC, including GC and EGJ, is currently are treated with the same systemic regimens in the metastatic setting. Overall survival metastatic disease remains poor, with few molecular targeted approaches having been successfully incorporated into routine care to date - only first line anti-HER2 therapy for $E R B B 2$ amplification and second line anti-VEGFR2 therapy have succeeded in improving outcomes. Molecular characterization of GEC has identified other relatively frequent mutations and copy number variations in EGFR, FGFR2, MET, PI3K, and other oncogenes, candidate biomarkers, and immuno-oncologic checkpoints that may serve as actionable therapeutic targets. Here we review these key aberrations, their impact on protein expression, therapeutic implications, and potential future clinical directions within each pathway.

\section{Keywords}

Gastric cancer; esophagogastric junction cancer; gastroesophageal adenocarcinoma; HER2;

VEGFR2; EGFR; MET; FGFR2; claudin 18.2

\section{Background}

Distal gastric adenocarcinoma (GC) is the fifth most common cancer globally and third most common cause of cancer-related mortality. ${ }^{1-3}$ Approximately twenty-five thousand new GC cases and eleven thousand deaths occurred in the United States in $2015 .{ }^{4}$ Esophagogastric junction adenocarcinoma (EGJ) continues to increase in incidence. For both diseases, the

Corresponding Author: Daniel V.T. Catenacci, MD, University of Chicago Comprehensive Cancer Center, Section of Hematology/ Oncology, 5841 S. Maryland Avenue, Chicago, IL 60637, dcatenac@ medicine.bsd.uchicago.edu.

Publisher's Disclaimer: This is a PDF file of an unedited manuscript that has been accepted for publication. As a service to our customers we are providing this early version of the manuscript. The manuscript will undergo copyediting, typesetting, and review of the resulting proof before it is published in its final citable form. Please note that during the production process errors may be discovered which could affect the content, and all legal disclaimers that apply to the journal pertain. 
majority of patients present with either metastatic or locally advanced disease, and even patients with earlier stage disease have a high risk of recurrence despite aggressive perioperative therapy. In the metastatic setting, the median overall survival is approximately 11 months with optimal palliative chemotherapy. Over the past decade, molecular subtyping of GEC has highlighted the inter-patient heterogeneity of GEC and uncovered potentially actionable molecular pathways. ${ }^{5}$ Routine next generation sequencing identified that at least $37 \%$ of GC patients harbor genetic alterations in receptor tyrosine kinases (RTKs), including ERBB2, MET, EGFR, KRAS, and FGFR2. ${ }^{6-8}$ These genomic events, as well as recently derived key subsets of the disease, namely microsatellite instability-high (MSI-high), EBVassociated (EBV), chromosomal instability (CIN), and genomically stable (GS), provide for more specific molecularly targeted therapeutic possibilities. ${ }^{9}$

\section{ERBB2}

ERBB2, or HER2, is a transmembrane RTK within the epidermal growth factor receptor (EGFR) family, encoded on chromosome17q21. HER2 regulates proliferation, adhesion, differentiation, and migration via activation of the RAS-MAPK and PI3K-AKT pathways. HER2 lacks an exogenous ligand and is transactivated via heterodimerization with other HER family members leading to downstream kinase activation. Significant and therapeutically relevant over-expression results predominantly from gene amplification. HER2 IHC expression localizes to the cell membrane in well-differentiated adenocarcinoma and to the cytoplasm in poorly-differentiated adenocarcinomas, which may affect treatment response. ${ }^{10}$ HER2 expressing tumors are more common with EGJ (15-20\%) compared with the distal GC (10-15\%), and the prognostic impact of HER2 expression remains controversial. ${ }^{11-18}$

Effective targeting of HER2 in gastric cancer was initially demonstrated using trastuzumab, a humanized monoclonal anti-HER2 antibody against the HER2 ectodomain (Table 1). The phase III Trastuzumab for Gastric Cancer (ToGA) trial evaluated first line fluoropyrimidine/ cisplatin chemotherapy doublet with or without trastuzumab in patients with HER-2 overexpressing (any IHC 3+ or FISH HER2:CEP17 ratio 22) unresectable or metastatic GEC. Patients receiving trastuzumab survived a median of 13.8 months versus 11.1 months with chemotherapy alone, and response rates were $47 \%$ and $35 \%$ respectively in the intention-totreat (ITT) population. In a subset analysis, median survival was 16 vs 11.8 months in the combined IHC2+/FISH+ and IHC3+ groups, accounting for $77 \%$ of the patients. This trial therefore led to the approval of trastuzumab in HER2 over-expressing gastric cancer for the $\mathrm{IHC} 2+/ \mathrm{FISH}+$ and IHC3+ subsets of the trial. ${ }^{18,19}$

Though HER-2 over-expression/ERBB2 amplification predicts benefit from the anti-HER2 antibody trastuzumab in the first line setting, ${ }^{18}$ the definition of positivity and trial inclusion criteria within trials has evolved over time. Current clinical diagnostic testing requires evaluation by a combination of IHC (membranous reactivity in $\geq 10 \%$ of cancer cells in a surgical specimen or a cluster of at least 5 cells in a biopsy specimen), and fluorescence insitu hybridization (FISH with HER2:CEP17 ratio $\geq 2$ ). IHC 0/1 is considered negative, and IHC3+ is considered positive without the need for FISH testing, while IHC2+ requires FISH assessment. Higher throughput assays, including mass spectrometry and next-generation 
sequencing (NGS), have emerged with the potential to refine diagnostic accuracy as well as possessing multiplexing capability to assess for other relevant aberrations. ${ }^{5,20,21}$ Assessment of ERBB2 amplification by cell free DNA (cfDNA) is also emerging as a potential noninvasive strategy, potentially for serial assessment of ERBB2 status over time in a noninvasive manner, ${ }^{22}$ given reports supporting the concept of tumor evolution including the potential loss of ERBB2 expression over time. ${ }^{21,23-25}$

Lapatinib, a selective intracellular tyrosine kinase inhibitor (TKI) of ERBB1 and EERBB2 was also studied in first and second line GEC (Table 1). The phase III TRIO-013/LOGiC (Lapatinib in Combination With Capecitabine Plus Oxaliplatin in Human Epidermal Growth Factor Receptor 2-Positive Advanced or Metastatic Gastric, Esophageal, or Gastroesophageal Adenocarcinoma) trial randomized 545 untreated HER2 positive (HER2:CEP17 ratio $\geq 2$ by FISH or IHC 3+ if FISH not available) GEC patients to receive capecitabine and oxaliplatin in addition to either lapatinib or placebo. Lapatinib increased objective response from 39\% to 53\%, and modestly increased median PFS from 5.4 to 6 months, but failed to confer an overall survival benefit in the ITT population. ${ }^{26}$ Younger and Asian patients appeared to derive the most benefit in subset analyses. The absolute level of amplification positively correlated with outcome, as previously described, ${ }^{27,28}$ signifying heterogeneity of benefit within the current HER2 positive classification. Recently, the degree of amplification has been shown to correlate closely with absolute protein expression level, again closely associated with clinical benefit. ${ }^{20,29}$ The variations in absolute amplification/ expression across various trials, as well as lack of antibody-dependent cell-mediated cytotoxicity (ADCC) with lapatinib as compared to trastuzumab, serve as two of many potential explanations when contrasting outcomes of ToGA and LOGiC. In the second line, the phase III Asian TyTAN trial enrolled patients regardless of HER2 expression (FISH $\geq 2$ were eligible), where $31 \%$ of patients enrolled were FISH+ and IHC $0 / 1+.{ }^{30}$ Patients received paclitaxel alone or in combination with lapatinib. Despite response rates of $27 \%$ versus 9\%, no significant PFS or OS benefit was demonstrated in the ITT population. Of note, when limiting the evaluation to only those patients with 3+ HER2 expression by IHC, median survival improved to 14 months from 7.6 months in this subgroup ( $p=0.0176$ ), and progression-free survival 5.6 versus 4.2 months $(p=0.0101)$. This highlights the need to improve patient selection for targeted therapies.

Trastuzumab emtansine (T-DM1), an antibody-drug conjugate that is approved in HER2 positive metastatic breast cancer, was studied in the second line GATSBY trial (Table 1), but this failed to support a response or survival benefit versus paclitaxel monotherapy. ${ }^{31}$ Possible explanations for this negative trial include intra-patient tumor heterogeneity recognized to be more frequent than observed in breast cancer, with HER2-negative clones not controlled by targeted cytotoxic therapy,. There is now recognition of possible conversion to a HER2 negative status after first line therapy, making archived samples, as used in GATSBY (and TyTAN), inadequate for selecting appropriate HER2+ patients in second line. ${ }^{21,23-25}$

Whereas trastuzumab binds domain IV of HER2, the HER2- targeted agent pertuzumab binds domain II and thereby prevents dimerization. The CLEOPATRA trial revealed progression-free and overall survival benefits with the addition of pertuzumab to trastuzumab and chemotherapy in breast cancer, ${ }^{32}$ and initial results from the JACOB trial, 
evaluating pertuzumab in combination with trastuzumab and chemotherapy in the first line treatment of HER positive GEJ cancer, are eagerly awaited.

Thus, to date, no standard anti-HER2 directed approaches are recognized in trastuzumab refractory HER2+ GEC. However, standard chemotherapy with irinotecan or taxane based regimens are recommended. Notably, while second line ramucirumab trials, discussed below, included HER2 positive and trastuzumab-treated patients, this accounted for only $\sim 6 \%$ of patients enrolled in RAINBOW (Ramucirumab plus paclitaxel versus placebo plus paclitaxel in patients with previously treated advanced gastric or gastro-oesophageal junction adenocarcinoma) and $<1 \%$ of REGARD (Ramucirumab monotherapy for previously treated advanced gastric or gastro-oesophageal junction adenocarcinoma). ${ }^{33,34}$ Other strategies under evaluation in second and later lines of therapy include novel TKIs like apatinib, ${ }^{35}$ trastuzumab beyond progression, ${ }^{23,25}$ novel HER2 antibodies, ${ }^{36}$ and combination therapy with immune checkpoint inhibitors (Table 5). ${ }^{37}$

\section{VEGF}

Vascular endothelial growth factor (VEGF) induces angiogenesis and neovascularization. Over-expression is found in up to $58 \%$ of resected gastric cancer patients, ${ }^{38}$ and $7 \%$ of the Cancer Genome Atlas (TCGA) gastric cancer patients have VEGF-A amplification. ${ }^{9}$ Ramucirumab, a human IgG1 monoclonal antibody directly binding to VEGFR2 thus inhibiting VEGF ligand binding, was evaluated in the second line setting in the phase III REGARD trial (Table 2). ${ }^{34}$ Compared to supportive care, ramucirumab demonstrated an overall survival of 5.2 vs 3.8 months ( $p=0.047$ ), despite a response rate of only 3 percent the same as the placebo control. ${ }^{34}$ The phase III RAINBOW trial subsequently compared paclitaxel with or without ramucirumab in the second line setting (Table 2). Patients had a significant overall survival benefit of 9.6 vs 7.4 months $(p=0.017)$ as well as statistically significant PFS and ORR benefits. ${ }^{33}$ In the third line setting, apatinib, a multi-TKI, including VEGFR kinases, demonstrated an absolute 0.8 month PFS advantage (2.6 vs $1.8 \mathrm{mo}$ ) as well as a nearly 2 month overall survival benefit when compared with placebo in a Chinese population. ${ }^{39}$

Unfortunately, evaluation of anti-angiogenesis in the first line setting of GEC has been disappointing to date. The AVAGAST (Avastin in Gastric Cancer) trial evaluated chemotherapy (cisplatin and capecitabine) with or without bevacizumab, a humanized IgG1 VEGFA monoclonal antibody. Despite PFS (6.7 vs 5.3 months, $\mathrm{p}=0.0037$ ) and ORR (46\% vs $37.4 \%, \mathrm{p}=0.0315$ ) benefits, the improved median OS of 12.1 over 10.1 months observed in the control was not statistically significant. ${ }^{40}$ An Asian trial, AVATAR, confirmed these negative findings. ${ }^{41}$ A phase II trial of first line Ziv-aflibercept with FOLFOX recently did not meet its primary endpoint of 6 month PFS compared to FOLFOX alone. ${ }^{42}$ Additionally, a phase II trial in first line GEC comparing FOLFOX with or without ramucirumab was negative. ${ }^{43}$ Despite this, the phase III RAINFALL (Ramucirumab in Combination With Capecitabine and Cisplatin in Participants With Stomach Cancer) trial is currently evaluating first line ramucirumab/placebo in combination with capecitabine and cisplatin in metastatic gastric cancer, with results eagerly awaited (NCT02314117). 
Potential predictive biomarkers of response to anti-angiogenesis agents, to incorporate into routine practice have remained elusive. ${ }^{44}$ The AVAGAST trial suggested that patients with high plasma VEGF-A levels had increased overall survival compared with those expressing low VEGF-A levels, ${ }^{12}$ and hypertension as a biomarker has been noted. ${ }^{42}$ Further biomarker development may help identify a subset of patients that derive the most benefit from targeting this axis. However, biomarkers of response may remain undefined, potentially due to a marginal benefit realized by most patients.

\section{EGFR}

Epidermal growth factor receptor (EGFR) or ErbB1 is a transmembrane receptor and a wellrecognized mediator of oncogenic phenotype that is 'overexpressed' in approximately $30 \%$ of $\mathrm{GEC}^{45,46}$. EGFR-overexpressing tumors are associated with higher stage, more poorly differentiated histology, increased vascular invasion, and potentially shorter survival. ${ }^{14,47}$ EGFR amplification is found in only $\sim 5 \%$ of patients. $9,48,49$

EGFR-directed therapies evaluated in GEC include monoclonal antibodies such as cetuximab and panitumumab, which antagonize the extracellular binding domain. Preclinical data also suggested that cetuximab, a recombinant human-murine chimeric monoclonal antibody of a murine Fv region and a human IgG1 heavy and k light chain Fc region, induces ADCC. ${ }^{50}$ Small molecule TKIs, such as gefitinib, erlotinib, lapatinib, and afatinib competitively bind intracellularly to the tyrosine kinase domain. Early phase II trials combining cetuximab, panitumumab, or erlotinib with cytotoxic chemotherapy in GEC reported first line therapy response rates ranging from $41-65 \% .51-54$ Second line phase II evaluation of gefitinib or erlotinib monotherapy led to more modest responses of $\sim 9-11 \%$, and responses appeared limited to proximal EGJ cancers rather than distal GC. ${ }^{55,56}$

Subsequent phase III GEC trials targeting EGFR included EXPAND ((cetuximab, first line), REAL-2 (panitumumab, first line), and COG (gefitinib, second line) (Table 3). ${ }^{15,57,58}$ Each trial was negative, and panitumumab actually resulted in worse survival when combined with chemotherapy compared to chemotherapy alone. Notably, each of these trials enrolled all-comers without biomarker selection of any kind.

Xenograft models evaluating anti-EGFR therapy for EGFR-amplified tumors have reported potential benefit. ${ }^{59}$ In the phase II study combining FOLFOX with cetuximab, $22 \%$ of patients had greater than 4 EGFR copies, which correlated with increased overall survival. ${ }^{60}$ Similarly, in TRANS-COG, $15.6 \%$ of patients had increased gene copy number (GCN) including true EGFR amplification ( $5 \%)$; this latter small subset of of EGFR amplified patients derived a statistically significant survival benefit with the addition of gefitinib (HR $0.19, \mathrm{p}=0.007$ ). The EXPAND trial also demonstrated survival benefit in the small subset with extremely high EGFR expression by IHC H-Score (likely representing EGFR amplified tumors, but yet to be confirmed). ${ }^{61}$ With these recent promising subset analyses of EGFR amplification and consequent overexpression, future studies assessing the benefits of antiEGFR therapy in these patients are being pursued. ${ }^{62}$ Also, a Phase III trial of second line nimotuzumab with irinotecan (NCT01813253) is currently recruiting patients deemed to harbor EGFR overexpressing (IHC 2/3+) tumors (Table 5). 
The MET proto-oncogene encodes the c-MET receptor tyrosine kinase, which is involved in cell proliferation, angiogenesis, and migration. MET over-expression, as well as the subset with $M E T$ amplification, are each associated with worse survival in most reports. ${ }^{47,48,63-71}$ Canonical MET activation occurs via binding of its ligand, hepatocyte growth factor (HGF), but MET activation can also occur in an HGF-independent manner through RTK crosstalk. ${ }^{72,73}$ MET amplification leads to constitutive receptor activation independent of ligand, and is reported in $\sim 4-10 \%$ of GEC cases, ${ }^{48,74-76}$ but over-expression ranges from $23.7-70 \%$ in GEC, depending on the cohort evaluated and method of analysis used. ${ }^{66-68,77-79}$

As a predictive biomarker, early phase reports and trials initially suggested that GEC patients with MET-expressing tumors may benefit from MET-directed therapy. ${ }^{71,77}$ However, a subsequent phase II, ${ }^{80}$ and two Phase III MET-directed trials in GEC have been reported with overall negative results (Table 4). ${ }^{80,81}$ The MetGastric study evaluated onartuzumab, a humanized IgG1 antibody against the extracellular domain of c-MET, in combination with mFOLFOX6, in patients with c-MET-expressing tumors ( $\geq 1+, 250 \%$ cells). ${ }^{82}$ However, METGastric was terminated prematurely (70\% of planned accrual) due to negative results (in any MET expression group) in the prior/parallel YO28252 phase II biomarker evaluation trial of onartuzumab in unselected GEC patients. ${ }^{80}$ With this in mind, no benefit was seen in the ITT nor in the MET IHC $2 / 3+$ subgroup analysis ( $38 \%$ of enrolled patients, HR $0.64, \mathrm{p}=0.06$ ), which had less power to identify a benefit due to early termination of the trial. ${ }^{82}$ Similarly, RILOMET-1, which evaluated epirubicin, cisplatin, and capecitabine (ECX) with or without the addition of rilotumumab, a fully human IgG2 antibody against HGF ligand, was terminated due to an increased risk of death from the study drug. ${ }^{81}$ Ultimately, no improvement in any outcome was observed for the combination of rilotumumab and chemotherapy compared to chemotherapy alone.

One pitfall of these phase III trials is the loose definition of MET expression. In RILOMET-1, patients selection was defined as $\geq 1+$ MET expression by IHC in $\geq 25 \%$ of tumor cells to be eligible, and of all patients enrolled $\sim 90 \%$ were at the lowest $1+$ intensity of any extensity. Similarly, only $38 \%$ of METGastric were IHC 2/3+, yet as above, these patients demonstrated a near-significant benefit, in an under-accrued trial. Even with the large phase III MET inhibitor trials, one could argue that the selection for 'MET-dependent' cancers was too lenient and inadequate, and the highest expressing tumors underrepresented. ${ }^{48,74-76}$ Another negative trial in second line with the multi-targeted-TKI foretinib was observed, again without selecting patients by any biomarker. ${ }^{83}$

More promising results have been reported in smaller trials of MET inhibitors for MET amplified patients (4-5\% of GEC), ${ }^{48,67,74,76}$ with consequent over-expression. ${ }^{74}$ AMG-337, a relatively highly selective MET TKI, demonstrated clinical responses in MET-amplified advanced GEC patients (ORR 50\%), but the phase II study has been on hold after the expansion phase of the trial (results not publically available) ${ }^{84}$ Similarly, half of $M E T$ amplified patients treated with crizotinib in a phase I expansion cohort experienced response, ${ }^{48}$ and $75 \%$ of MET amplified patients receiving ABT-700 monoclonal antibody monotherapy demonstrated an objective response. ${ }^{85}$ The challenge of molecular heterogeneity, ${ }^{23,86}$ particularly in the CIN subset of GEC, ${ }^{9}$ may account for lack of response 
and relative quickly developed resistance to MET-directed therapy of MET amplified GEC. Any future therapeutic attempts with MET inhibitors will likely be directed towards the small subset of MET amplified patients (Table 5). ${ }^{87}$

\section{FGFR2}

Fibroblast growth factor type 2 ( FGFR2) encodes a RTK that regulates cell angiogenesis and proliferation, and when highly expressed, portends a poor prognosis in both Western and Asian populations. ${ }^{88}$ FGFR 2 expression in GEC is primarily driven by gene copy number, with $4-10 \%$ of GEC demonstrating FGFR 2 amplification. ${ }^{6,88,89}$ FGFR2 amplification is associated with diffuse type GC. Dovitinib, a TKI targeting FGFR 1-3, VEGF 1-3, PDGFR, and c-KIT, had promising results in in vitro studies. ${ }^{6}$ The SHINE trial compared paclitaxel with AZD4547, an FGFR2 TKI, in advanced GC in Asia, but the paclitaxel arm had superior survival - even in the FGFR2-amplified patient subset. ${ }^{90}$ On the other hand, another small phase IIa trial demonstrated response in 3/9 (33\%) with promising durability in FGFR2 amplified patients, and also observed that higher and homogenous gene amplification may better predict therapeutic benefit. ${ }^{91}$ These early phase trials suggest safety from this class of inhibitors, with mainly hypophosphatemia and ocular toxicities reported. ${ }^{90}$ Phase II trials are ongoing in combination with cytotoxic therapies and may be a more promising approach than monotherapy. Recently, 2/6 (33\%) FGFR2 over-expressing gastric cancer patients responded to FPA144, a novel humanized monoclonal IgG1 antibody against FGFR2b, in a chemotherapy-refractory phase I evaluation. ${ }^{92}$ Another $3 / 6$ (50\%) had stable disease, resulting in a 5/6 (83\%) disease control rate in this early assessment. Phase I expansion accrual remains ongoing in this patient population, with specific interest in FGFR2 amplified GEC patients (Table 5).

\section{PI3K-AKT-mTOR}

The PI3K-AKT-mTOR pathway is commonly altered in GEC leading to increased proliferation and apoptotic resistance, and this pathway is downstream from transmembrane receptors including HER2, EGFR, VEGFr, and FGFR. PIK3CA activating mutations, loss of PTEN, RICTOR amplification or AKT amplification have all been described. ${ }^{5,9,49}$ PIK3CA activating mutation alone is observed in $\sim 15 \%$ of GEC.

Attempted inhibition of mTOR with everolimus demonstrated a median PFS of 2.7 months and OS of 10.1 months in an early study, but the subsequent phase III GRANITE- 1 trial comparing everolimus with placebo in an unselected patient population as $2^{\text {nd }}$ or $3^{\text {rd }}$ line therapy failed to demonstrate an overall survival benefit. ${ }^{93,94}$ Results from a phase III trial of paclitaxel with or without everolimus are still awaited (NCT01248403). Numerous PI3K inhibitors have shown benefit in preclinical studies including BEZ235 and BKM120 as monotherapy, or in combination with HSP90 or hedgehog inhibitors, but the as yet uncertain clinical benefit in patients suggests a disconnect between preclinical models and treated GEC patients. ${ }^{95,96}$ Second line AKT inhibition trials are ongoing with AZD5363 in combination with paclitaxel (NCT02451956/NCT02449655), as well as the first line JAGUAR trial combining FOLFOX with or without AKT inhibition using ipatasertib (NCT01896531). Strategies selecting for only those with tumors having a genomically 
activated PI3K-AKT-mTOR pathway may enrich for clinical benefit with these targeted therapies (Table 5).

\section{CLDN18.2}

Claudins are structural components of tight junctions that seal the intercellular space, and are over-expressed in numerous cancers. CLDN18 gene amplification is found in 3\% of TCGA gastric cancer patients and 3\% of patients also harbor oncogenic gene fusions between $C L D N 18$ and $A R H G A P 26$, a RHOA inhibitor. ${ }^{97}$ These fusions impair cellextracellular membrane adhesion, and in doing so promote migration. Claudiximab (IMAB362) is a chimeric IgG1 monoclonal antibody against CLDN18.2 that is intended to enhance T-cell infiltration and ADCC. ${ }^{98}$ Claudiximab was developed from high throughput screening of malignancy-specific cell surface molecules that identified gastric specificity of the claudin 18 splice variant $2 .{ }^{99}$ Phase II evaluation in combination with EOX first line cytotoxic therapy demonstrated a 39\% ORR along with benefits in PFS and OS, most pronounced in high expressers of CLDN18.2 (Table 5). ${ }^{100}$ Further evaluation is planned in a phase III study.

\section{PD-L1}

Immune checkpoint inhibition with programmed-death 1 (PD-1) and programmed-death ligand 1 (PD-L1) inhibitors are under evaluation in many cancers, including GEC. PD-L1 normally binds to receptors on T-lymphocytes thereby inhibiting T-cell proliferation and inducing apoptosis, which impairs the cytotoxic immune response. EBV-associated GEC over-expresses PD-L1 and PD-L2 due to amplification of 9p24, ${ }^{101}$ and MSI-High tumors are also associated with intense immune infiltration and overexpression of checkpoint inhibitor targets. ${ }^{9,102}$ Keynote-012 evaluated pembrolizumab, a PD-1 inhibitor, in PDL1+ ( 30-40\% of patients screened) advanced GC, and demonstrated a $22 \%$ objective response rate. ${ }^{103}$ Of note, although PD-L1 over-expression correlated with a higher response rate, PD-L1 expression remains a poor predictive biomarker - patients without PD-L1 expression can still respond, and often those with expression do not derive benefit. ${ }^{103}$ Similarly, in the CheckMate-032 trial, objective response to nivolumab, another PD-1 inhibitor, was $18 \%$ and $12 \%$ in PD-L1 positive and negative patients, respectively in chemotherapy refractory GEC patients. ${ }^{104}$ Second line response rates, stratified by PD-L1 status, were similar in the Javelin GEC trial with avelumab, a PD-L1 antagonist (18.2\% vs 9.1\%), and less in later therapy lines (10\% vs $3.1 \%) .{ }^{105}$ RNA expression signatures reflecting interferon gamma activity and T-cell activity, which may identify patients more response to immune checkpoint inhibition, are currently under development to improve patient selection. ${ }^{106}$ Phase III evaluation of pembrolizumab (KEYNOTE-062, KEYNOTE-061) in the first line and second line metastatic settings respectively, and nivolumab (CheckMate-577) in the adjuvant setting are ongoing, with various other approaches in all lines of therapy being assessed with the various available checkpoint inhibitors. ${ }^{107}$

\section{CTLA-4}

Cytotoxic T lymphocyte antigen 4 (CTLA4) is constitutively expressed on the surface of Tregulatory cells and expression can be inducible on activated $\mathrm{T}$ lymphocytes and monocytes. Up-regulation leads to IL-2 production and IL-2R expression, with subsequent priming of 
the T-cell response ${ }^{108}$. Mutations and copy number variation of CTLA4 occur rarely in only $2.5 \%$ of TCGA gastric cancer patients. Tremelimumab is a fully humanized IgG2 antiCTLA4 monoclonal antibody that has undergone evaluation in numerous other malignancies. Of the 18 metastatic GC patients enrolled in a second line phase II trial, no objective responses were seen after cycle 1 . However, 12-month survival was $33 \%$, and one patient later derived a partial response after 25.4 months of therapy and was alive at 32.7 months at the time of publication ${ }^{109}$. Ipilimumab, another CTLA-4 antagonist, was evaluated as maintenance therapy after receiving a platinum fluoropyrimidine doublet, but failed to prolong PFS or OS. ${ }^{110}$ Evaluation remains underway in combination with PD-1/PD-L1 antagonists as discussed above. ${ }^{107}$

\section{Summary}

Despite trials evaluating targeted therapies for GEC, only trastuzumab and ramucirumab have demonstrated benefit and been approved in the first and second line settings, respectively. Subset analyses have successively identified patients with MET, EGFR and FGFR2 genomic aberrations that may benefit from matched targeted therapies, yet designing traditional trials for such infrequent aberrations and inter-patient heterogeneity continues to remain extremely challenging. Tumor molecular heterogeneity within patients and over time and serial lines of therapy, are also hurdles to successful implementation of these targeted agents. Novel trial designs such as the PANGEA type II expansion platform may better identify and treat these uncommon actionable aberrations as a way forward by testing a treatment strategy. ${ }^{23,62,111}$ Immune checkpoint inhibitors are being evaluated and hold promise, with investigations ongoing to determine subsets likely to derive benefit. Future directions should focus on improving diagnostic assays and validating predictive biomarkers for benefit from immuno-oncologic therapies.

\section{Acknowledgments}

Disclosure statement

Dr. Catenacci has received research funding from Genentech/Roche, Amgen, OncoplexDx/Nantomics and honoraria from Genentech/Roche, Amgen, Eli Lilly, Five Prime, OncoplexDx/Nantomics, Guardant Health, Foundation Medicine. Dr. Maron has nothing to disclose.

\section{References}

1. Torre LA, Bray F, Siegel RL, Ferlay J, Lortet-Tieulent J, Jemal A. Global cancer statistics, 2012. CA Cancer J Clin. 2015; 65(2):87-108. [PubMed: 25651787]

2. Sehdev A, Catenacci DV. Gastroesophageal cancer: focus on epidemiology, classification, and staging. Discov Med. 2013; 16(87):103-111. [PubMed: 23998446]

3. Sehdev A, Catenacci DV. Perioperative therapy for locally advanced gastroesophageal cancer: current controversies and consensus of care. J Hematol Oncol. 2013; 6:66. [PubMed: 24010946]

4. Siegel RL, Miller KD, Jemal A. Cancer statistics, 2015. CA Cancer J Clin. 2015; 65(1):5-29. [PubMed: 25559415]

5. Ali SM, Sanford EM, Klempner SJ, et al. Prospective comprehensive genomic profiling of advanced gastric carcinoma cases reveals frequent clinically relevant genomic alterations and new routes for targeted therapies. Oncologist. 2015; 20(5):499-507. [PubMed: 25882375] 
6. Deng N, Goh LK, Wang H, et al. A comprehensive survey of genomic alterations in gastric cancer reveals systematic patterns of molecular exclusivity and co-occurrence among distinct therapeutic targets. Gut. 2012; 61(5):673-684. [PubMed: 22315472]

7. Stachler MD, Taylor-Weiner A, Peng S, et al. Paired exome analysis of Barrett's esophagus and adenocarcinoma. Nat Genet. 2015; 47(9):1047-1055. [PubMed: 26192918]

8. Zang ZJ, Ong CK, Cutcutache I, et al. Genetic and structural variation in the gastric cancer kinome revealed through targeted deep sequencing. Cancer Res. 2011; 71(1):29-39. [PubMed: 21097718]

9. Cancer Genome Atlas Research N. Comprehensive molecular characterization of gastric adenocarcinoma. Nature. 2014; 513(7517):202-209. [PubMed: 25079317]

10. Kameda T, Yasui W, Yoshida K, et al. Expression of ERBB2 in human gastric carcinomas: relationship between p185ERBB2 expression and the gene amplification. Cancer Res. 1990; 50(24):8002-8009. [PubMed: 1979253]

11. Okines AF, Thompson LC, Cunningham D, et al. Effect of HER2 on prognosis and benefit from peri-operative chemotherapy in early oesophago-gastric adenocarcinoma in the MAGIC trial. Ann Oncol. 2013; 24(5):1253-1261. [PubMed: 23233651]

12. Van Cutsem E, de Haas S, Kang YK, et al. Bevacizumab in combination with chemotherapy as first-line therapy in advanced gastric cancer: a biomarker evaluation from the AVAGAST randomized phase III trial. J Clin Oncol. 2012; 30(17):2119-2127. [PubMed: 22565005]

13. Gordon MA, Gundacker HM, Benedetti J, et al. Assessment of HER2 gene amplification in adenocarcinomas of the stomach or gastroesophageal junction in the INT-0116/SWOG9008 clinical trial. Ann Oncol. 2013; 24(7):1754-1761. [PubMed: 23524864]

14. Terashima M, Kitada K, Ochiai A, et al. Impact of expression of human epidermal growth factor receptors EGFR and ERBB2 on survival in stage II/III gastric cancer. Clin Cancer Res. 2012; 18(21):5992-6000. [PubMed: 22977193]

15. Lordick F, Kang YK, Chung HC, et al. Capecitabine and cisplatin with or without cetuximab for patients with previously untreated advanced gastric cancer (EXPAND): a randomised, open-label phase 3 trial. Lancet Oncol. 2013; 14(6):490-499. [PubMed: 23594786]

16. Waddell T, Chau I, Cunningham D, et al. Epirubicin, oxaliplatin, and capecitabine with or without panitumumab for patients with previously untreated advanced oesophagogastric cancer (REAL3): a randomised, open-label phase 3 trial. Lancet Oncol. 2013; 14(6):481-489. [PubMed: 23594787]

17. Kurokawa Y, Matsuura N, Kimura Y, et al. Multicenter large-scale study of prognostic impact of HER2 expression in patients with resectable gastric cancer. Gastric Cancer. 2015; 18(4):691-697. [PubMed: 25224659]

18. Bang YJ, Van Cutsem E, Feyereislova A, et al. Trastuzumab in combination with chemotherapy versus chemotherapy alone for treatment of HER2-positive advanced gastric or gastro-oesophageal junction cancer (ToGA): a phase 3, open-label, randomised controlled trial. Lancet. 2010; 376(9742):687-697. [PubMed: 20728210]

19. Hofmann M, Stoss O, Shi D, et al. Assessment of a HER2 scoring system for gastric cancer: results from a validation study. Histopathology. 2008; 52(7):797-805. [PubMed: 18422971]

20. Catenacci DV, Liao WL, Zhao L, et al. Mass-spectrometry-based quantitation of Her2 in gastroesophageal tumor tissue: comparison to IHC and FISH. Gastric Cancer. 2015

21. Sellappan S, Blackler A, Liao WL, et al. Therapeutically Induced Changes in HER2, HER3, and EGFR Protein Expression for Treatment Guidance. J Natl Compr Canc Netw. 2016; 14(5):503507. [PubMed: 27160229]

22. Shoda K, Masuda K, Ichikawa D, et al. HER2 amplification detected in the circulating DNA of patients with gastric cancer: a retrospective pilot study. Gastric Cancer. 2015; 18(4):698-710. [PubMed: 25322965]

23. Catenacci DV. Next-generation clinical trials: Novel strategies to address the challenge of tumor molecular heterogeneity. Mol Oncol. 2015; 9(5):967-996. [PubMed: 25557400]

24. Janjigian YY, Riches JC, Ku GY, et al. Loss of human epidermal growth factor receptor 2 (HER2) expression in HER2-overexpressing esophagogastric (EG) tumors treated with trastuzumab. Paper presented at: ASCO Annual Meeting Proceedings. 2015 
25. Denlinger CS, Alsina Maqueda M, Watkins DJ, et al. Randomized phase 2 study of paclitaxel (PTX), trastuzumab (T) with or without MM-111 in HER2 expressing gastroesophageal cancers (GEC). Paper presented at: ASCO Annual Meeting Proceedings. 2016

26. Hecht JR, Bang YJ, Qin SK, et al. Lapatinib in Combination With Capecitabine Plus Oxaliplatin in Human Epidermal Growth Factor Receptor 2-Positive Advanced or Metastatic Gastric, Esophageal, or Gastroesophageal Adenocarcinoma: TRIO-013/LOGiC-A Randomized Phase III Trial. J Clin Oncol. 2016; 34(5):443-451. [PubMed: 26628478]

27. Gomez-Martin C, Plaza JC, Pazo-Cid R, et al. Level of HER2 gene amplification predicts response and overall survival in HER2-positive advanced gastric cancer treated with trastuzumab. J Clin Oncol. 2013; 31(35):4445-4452. [PubMed: 24127447]

28. Ock CY, Lee KW, Kim JW, et al. Optimal Patient Selection for Trastuzumab Treatment in HER2Positive Advanced Gastric Cancer. Clin Cancer Res. 2015; 21(11):2520-2529. [PubMed: 25712681]

29. An E, Ock C-Y, Kim T-Y, et al. Clinical value of proteomic HER2 assessment for patients with gastric cancer. Annals of Oncology. 2016

30. Satoh T, Xu RH, Chung HC, et al. Lapatinib plus paclitaxel versus paclitaxel alone in the secondline treatment of HER2-amplified advanced gastric cancer in Asian populations: TyTAN-a randomized, phase III study. J Clin Oncol. 2014; 32(19):2039-2049. [PubMed: 24868024]

31. Kang Y-K, Shah MA, Ohtsu A, et al. A randomized, open-label, multicenter, adaptive phase $2 / 3$ study of trastuzumab emtansine (T-DM1) versus a taxane (TAX) in patients (pts) with previously treated HER2-positive locally advanced or metastatic gastric/gastroesophageal junction adenocarcinoma (LA/MGC/GEJC). Paper presented at: ASCO Annual Meeting Proceedings. 2016

32. Swain SM, Kim SB, Cortes J, et al. Pertuzumab, trastuzumab, and docetaxel for HER2-positive metastatic breast cancer (CLEOPATRA study): overall survival results from a randomised, doubleblind, placebo-controlled, phase 3 study. Lancet Oncol. 2013; 14(6):461-471. [PubMed: 23602601]

33. Wilke H, Muro K, Van Cutsem E, et al. Ramucirumab plus paclitaxel versus placebo plus paclitaxel in patients with previously treated advanced gastric or gastro-oesophageal junction adenocarcinoma (RAINBOW): a double-blind, randomised phase 3 trial. Lancet Oncol. 2014; 15(11):1224-1235. [PubMed: 25240821]

34. Fuchs CS, Tomasek J, Yong CJ, et al. Ramucirumab monotherapy for previously treated advanced gastric or gastro-oesophageal junction adenocarcinoma (REGARD): an international, randomised, multicentre, placebo-controlled, phase 3 trial. Lancet. 2014; 383(9911):31-39. [PubMed: 24094768]

35. Janjigian YY, Capanu M, Imtiaz T, et al. A phase II study of afatinib in patients (pts) with metastatic human epidermal growth factor receptor (HER2)-positive trastuzumab-refractory esophagogastric (EG) cancer. Paper presented at: ASCO Annual Meeting Proceedings. 2014

36. Rugo HS, Pegram MD, Gradishar WJ, et al. SOPHIA: A phase 3, randomized study of margetuximab (M) plus chemotherapy (CTX) vs trastuzumab (T) plus CTX in the treatment of patients with HER2+ metastatic breast cancer (MBC). ASCO Meeting Abstracts. 2016; 34(15_suppl):TPS630.

37. Combination Margetuximab and Pembrolizumab for Advanced, Metastatic HER2(+) Gastric or Gastroesophageal Junction Cancer. https://ClinicalTrials.gov/show/NCT02689284

38. Oh SY, Kwon HC, Kim SH, et al. Clinicopathologic significance of HIF-1alpha, p53, and VEGF expression and preoperative serum VEGF level in gastric cancer. BMC Cancer. 2008; 8:123. [PubMed: 18452596]

39. Li J, Qin S, Xu J, et al. Randomized, Double-Blind, Placebo-Controlled Phase III Trial of Apatinib in Patients With Chemotherapy-Refractory Advanced or Metastatic Adenocarcinoma of the Stomach or Gastroesophageal Junction. J Clin Oncol. 2016; 34(13):1448-1454. [PubMed: 26884585]

40. Ohtsu A, Shah MA, Van Cutsem E, et al. Bevacizumab in combination with chemotherapy as firstline therapy in advanced gastric cancer: a randomized, double-blind, placebo-controlled phase III study. J Clin Oncol. 2011; 29(30):3968-3976. [PubMed: 21844504] 
41. Shen L, Li J, Xu J, et al. Bevacizumab plus capecitabine and cisplatin in Chinese patients with inoperable locally advanced or metastatic gastric or gastroesophageal junction cancer: randomized, double-blind, phase III study (AVATAR study). Gastric Cancer. 2015; 18(1):168-176. [PubMed: 24557418]

42. Enzinger PC, McCleary NJ, Zheng H, et al. Multicenter double-blind randomized phase II: FOLFOX+ ziv-aflibercept/placebo for patients (pts) with chemo-naive metastatic esophagogastric adenocarcinoma (MEGA). Paper presented at: ASCO Annual Meeting Proceedings. 2016

43. Yoon HH, Bendell JC, Braiteh FS, et al. Ramucirumab (RAM) plus FOLFOX as front-line therapy $(\mathrm{Rx})$ for advanced gastric or esophageal adenocarcinoma (GE-AC): randomized, double-blind, multicenter phase 2 trial. Paper presented at: ASCO Annual Meeting Proceedings. 2014

44. Murukesh N, Dive C, Jayson GC. Biomarkers of angiogenesis and their role in the development of VEGF inhibitors. Br J Cancer. 2010; 102(1):8-18. [PubMed: 20010945]

45. Kim MA, Lee HS, Lee HE, Jeon YK, Yang HK, Kim WH. EGFR in gastric carcinomas: prognostic significance of protein overexpression and high gene copy number. Histopathology. 2008; 52(6): 738-746. [PubMed: 18397279]

46. Wang KL, Wu TT, Choi IS, et al. Expression of epidermal growth factor receptor in esophageal and esophagogastric junction adenocarcinomas: association with poor outcome. Cancer. 2007; 109(4): 658-667. [PubMed: 17211865]

47. Nagatsuma AK, Aizawa M, Kuwata T, et al. Expression profiles of HER2, EGFR, MET and FGFR2 in a large cohort of patients with gastric adenocarcinoma. Gastric Cancer. 2015; 18(2): 227-238. [PubMed: 24626858]

48. Lennerz JK, Kwak EL, Ackerman A, et al. MET amplification identifies a small and aggressive subgroup of esophagogastric adenocarcinoma with evidence of responsiveness to crizotinib. J Clin Oncol. 2011; 29(36):4803-4810. [PubMed: 22042947]

49. Dulak AM, Stojanov P, Peng S, et al. Exome and whole-genome sequencing of esophageal adenocarcinoma identifies recurrent driver events and mutational complexity. Nat Genet. 2013; 45(5):478-486. [PubMed: 23525077]

50. Kimura H, Sakai K, Arao T, Shimoyama T, Tamura T, Nishio K. Antibody-dependent cellular cytotoxicity of cetuximab against tumor cells with wild-type or mutant epidermal growth factor receptor. Cancer Sci. 2007; 98(8):1275-1280. [PubMed: 17498200]

51. Enzinger PC, Burtness BA, Niedzwiecki D, et al. CALGB 80403 (Alliance)/E1206: A Randomized Phase II Study of Three Chemotherapy Regimens Plus Cetuximab in Metastatic Esophageal and Gastroesophageal Junction Cancers. J Clin Oncol. 2016

52. Wainberg ZA, Lin LS, DiCarlo B, et al. Phase II trial of modified FOLFOX6 and erlotinib in patients with metastatic or advanced adenocarcinoma of the oesophagus and gastro-oesophageal junction. Br J Cancer. 2011; 105(6):760-765. [PubMed: 21811258]

53. Lordick F, Luber B, Lorenzen S, et al. Cetuximab plus oxaliplatin/leucovorin/5-fluorouracil in first-line metastatic gastric cancer: a phase II study of the Arbeitsgemeinschaft Internistische Onkologie (AIO). Br J Cancer. 2010; 102(3):500-505. [PubMed: 20068568]

54. Tebbutt NC, Price TJ, Ferraro DA, et al. Panitumumab added to docetaxel, cisplatin and fluoropyrimidine in oesophagogastric cancer: ATTAX3 phase II trial. Br J Cancer. 2016; 114(5): 505-509. [PubMed: 26867157]

55. Ferry DR, Anderson M, Beddard K, et al. A phase II study of gefitinib monotherapy in advanced esophageal adenocarcinoma: evidence of gene expression, cellular, and clinical response. Clin Cancer Res. 2007; 13(19):5869-5875. [PubMed: 17908981]

56. Dragovich T, McCoy S, Fenoglio-Preiser CM, et al. Phase II trial of erlotinib in gastroesophageal junction and gastric adenocarcinomas: SWOG 0127. J Clin Oncol. 2006; 24(30):4922-4927. [PubMed: 17050876]

57. Okines AF, Ashley SE, Cunningham D, et al. Epirubicin, oxaliplatin, and capecitabine with or without panitumumab for advanced esophagogastric cancer: dose-finding study for the prospective multicenter, randomized, phase II/III REAL-3 trial. J Clin Oncol. 2010; 28(25):3945-3950. [PubMed: 20679619] 
58. Dutton SJ, Ferry DR, Blazeby JM, et al. Gefitinib for oesophageal cancer progressing after chemotherapy (COG): a phase 3, multicentre, double-blind, placebo-controlled randomised trial. Lancet Oncol. 2014; 15(8):894-904. [PubMed: 24950987]

59. Zhang L, Yang J, Cai J, et al. A subset of gastric cancers with EGFR amplification and overexpression respond to cetuximab therapy. Sci Rep. 2013; 3:2992. [PubMed: 24141978]

60. Luber B, Deplazes J, Keller G, et al. Biomarker analysis of cetuximab plus oxaliplatin/ leucovorin/5-fluorouracil in first-line metastatic gastric and oesophago-gastric junction cancer: results from a phase II trial of the Arbeitsgemeinschaft Internistische Onkologie (AIO). BMC Cancer. 2011; 11:509. [PubMed: 22152101]

61. Lordick F, Kang Y-K, Salman P, et al. Clinical outcome according to tumor HER2 status and EGFR expression in advanced gastric cancer patients from the EXPAND study. Paper presented at: ASCO Annual Meeting Proceedings. 2013

62. Catenacci DVT, Polite BN, Henderson L, et al. Toward personalized treatment for gastroesophageal adenocarcinoma (GEC): Strategies to address tumor heterogeneity-PANGEA. Paper presented at: ASCO Annual Meeting Proceedings. 2014

63. Catenacci DVT, Tang R, Oliner KS, et al. MET as a prognostic biomarker of survival in a large cohort of patients with gastroesophageal cancer (GEC). Paper presented at: ASCO Annual Meeting Proceedings. 2015

64. Hack SP, Bruey JM, Koeppen H. HGF/MET-directed therapeutics in gastroesophageal cancer: a review of clinical and biomarker development. Oncotarget. 2014; 5(10):2866-2880. [PubMed: 24930887]

65. Metzger ML, Behrens HM, Boger C, Haag J, Kruger S, Rocken C. MET in gastric cancerdiscarding a 10\% cutoff rule. Histopathology. 2016; 68(2):241-253. [PubMed: 26033401]

66. Nakajima M, Sawada H, Yamada Y, et al. The prognostic significance of amplification and overexpression of c-met and c-erb B-2 in human gastric carcinomas. Cancer. 1999; 85(9):18941902. [PubMed: 10223227]

67. Lee HE, Kim MA, Lee HS, et al. MET in gastric carcinomas: comparison between protein expression and gene copy number and impact on clinical outcome. Br J Cancer. 2012; 107(2):325333. [PubMed: 22644302]

68. Graziano F, Galluccio N, Lorenzini P, et al. Genetic activation of the MET pathway and prognosis of patients with high-risk, radically resected gastric cancer. J Clin Oncol. 2011; 29(36):4789-4795. [PubMed: 22042954]

69. Catenacci DV, Cervantes G, Yala S, et al. RON (MST1R) is a novel prognostic marker and therapeutic target for gastroesophageal adenocarcinoma. Cancer Biol Ther. 2011; 12(1):9-46. [PubMed: 21543897]

70. Yu S, Yu Y, Zhao N, Cui J, Li W, Liu T. C-Met as a prognostic marker in gastric cancer: a systematic review and meta-analysis. PLoS One. 2013; 8(11):e79137. [PubMed: 24223894]

71. Iveson T, Donehower RC, Davidenko I, et al. Rilotumumab in combination with epirubicin, cisplatin, and capecitabine as first-line treatment for gastric or oesophagogastric junction adenocarcinoma: an open-label, dose deescalation phase $1 \mathrm{~b}$ study and a double-blind, randomised phase 2 study. Lancet Oncol. 2014; 15(9):1007-1018. [PubMed: 24965569]

72. Jo M, Stolz DB, Esplen JE, Dorko K, Michalopoulos GK, Strom SC. Cross-talk between epidermal growth factor receptor and c-Met signal pathways in transformed cells. J Biol Chem. 2000; 275(12):8806-8811. [PubMed: 10722725]

73. Yamaguchi H, Chang SS, Hsu JL, Hung MC. Signaling cross-talk in the resistance to HER family receptor targeted therapy. Oncogene. 2014; 33(9):1073-1081. [PubMed: 23542173]

74. Catenacci DV, Liao WL, Thyparambil S, et al. Absolute quantitation of Met using mass spectrometry for clinical application: assay precision, stability, and correlation with MET gene amplification in FFPE tumor tissue. PLoS One. 2014; 9(7):e100586. [PubMed: 24983965]

75. Jardim DL, Tang C, Gagliato Dde M, et al. Analysis of 1,115 patients tested for MET amplification and therapy response in the MD Anderson Phase I Clinic. Clin Cancer Res. 2014; 20(24):63366345. [PubMed: 25326232] 
76. Smolen GA, Sordella R, Muir B, et al. Amplification of MET may identify a subset of cancers with extreme sensitivity to the selective tyrosine kinase inhibitor PHA-665752. Proc Natl Acad Sci U S A. 2006; 103(7):2316-2321. [PubMed: 16461907]

77. Catenacci DV, Henderson L, Xiao SY, et al. Durable complete response of metastatic gastric cancer with anti-Met therapy followed by resistance at recurrence. Cancer Discov. 2011; 1(7):573-579. [PubMed: 22389872]

78. Toiyama Y, Yasuda H, Saigusa S, et al. Co-expression of hepatocyte growth factor and c-Met predicts peritoneal dissemination established by autocrine hepatocyte growth factor/c-Met signaling in gastric cancer. Int J Cancer. 2012; 130(12):2912-2921. [PubMed: 21796631]

79. Zhao J, Zhang X, Xin Y. Up-regulated expression of Ezrin and c-Met proteins are related to the metastasis and prognosis of gastric carcinomas. Histol Histopathol. 2011; 26(9):1111-1120. [PubMed: 21751142]

80. Shah MA, Cho JY, Tan IB, et al. A Randomized Phase II Study of FOLFOX With or Without the MET Inhibitor Onartuzumab in Advanced Adenocarcinoma of the Stomach and Gastroesophageal Junction. Oncologist. 2016

81. Cunningham D, Tebbutt NC, Davidenko I, et al. Phase III, randomized, double-blind, multicenter, placebo $(\mathrm{P})$-controlled trial of rilotumumab $(\mathrm{R})$ plus epirubicin, cisplatin and capecitabine (ECX) as first-line therapy in patients (pts) with advanced MET-positive (pos) gastric or gastroesophageal junction (G/GEJ) cancer: RILOMET-1 study. Paper presented at: ASCO Annual Meeting Proceedings. 2015

82. Shah MA, Bang Y-J, Lordick F, et al. METGastric: A phase III study of onartuzumab plus mFOLFOX6 in patients with metastatic HER2-negative (HER2-) and MET-positive (MET+) adenocarcinoma of the stomach or gastroesophageal junction (GEC). Paper presented at: ASCO Annual Meeting Proceedings. 2015

83. Shah MA, Wainberg ZA, Catenacci DV, et al. Phase II study evaluating 2 dosing schedules of oral foretinib (GSK1363089), cMET/VEGFR2 inhibitor, in patients with metastatic gastric cancer. PLoS One. 2013; 8(3):e54014. [PubMed: 23516391]

84. Kwak EL, LoRusso P, Hamid O, et al. Clinical activity of AMG 337, an oral MET kinase inhibitor, in adult patients (pts) with MET-amplified gastroesophageal junction (GEJ), gastric (G), or esophageal (E) cancer. Paper presented at: ASCO Annual Meeting Proceedings. 2015

85. Kang Y-K, LoRusso P, Salgia R, et al. Phase I study of ABT-700, an anti-c-Met antibody, in patients (pts) with advanced gastric or esophageal cancer (GEC). Paper presented at: ASCO Annual Meeting Proceedings. 2015

86. Kwak EL, Ahronian LG, Siravegna G, et al. Molecular Heterogeneity and Receptor Coamplification Drive Resistance to Targeted Therapy in MET-Amplified Esophagogastric Cancer. Cancer Discov. 2015; 5(12):1271-1281. [PubMed: 26432108]

87. Mullard A. NCI-MATCH trial pushes cancer umbrella trial paradigm. Nat Rev Drug Discov. 2015; 14(8):513-515. [PubMed: 26228747]

88. Su X, Zhan P, Gavine PR, et al. FGFR2 amplification has prognostic significance in gastric cancer: results from a large international multicentre study. Br J Cancer. 2014; 110(4):967-975. [PubMed: 24457912]

89. Matsumoto K, Arao T, Hamaguchi T, et al. FGFR2 gene amplification and clinicopathological features in gastric cancer. Br J Cancer. 2012; 106(4):727-732. [PubMed: 22240789]

90. Bang Y-J, Van Cutsem E, Mansoor W, et al. A randomized, open-label phase II study of AZD4547 (AZD) versus Paclitaxel $(\mathrm{P})$ in previously treated patients with advanced gastric cancer (AGC) with Fibroblast Growth Factor Receptor 2 (FGFR2) polysomy or gene amplification (amp): SHINE study. Paper presented at: ASCO Annual Meeting Proceedings. 2015

91. Smyth EC, Turner NC, Pearson A, et al. Phase II study of AZD4547 in FGFR amplified tumours: Gastroesophageal cancer (GC) cohort pharmacodynamic and biomarker results. Paper presented at: ASCO Annual Meeting Proceedings. 2016

92. Lee J, Bendell JC, Rha SY, et al. Antitumor activity and safety of FPA144, an ADCC-enhanced, FGFR2b isoform-selective monoclonal antibody, in patients with FGFR2b+ gastric cancer and advanced solid tumors. ASCO Meeting Abstracts. 2016; 34(15_suppl):2502. 
93. Doi T, Muro K, Boku N, et al. Multicenter phase II study of everolimus in patients with previously treated metastatic gastric cancer. J Clin Oncol. 2010; 28(11):1904-1910. [PubMed: 20231677]

94. Ohtsu A, Ajani JA, Bai YX, et al. Everolimus for previously treated advanced gastric cancer: results of the randomized, double-blind, phase III GRANITE-1 study. J Clin Oncol. 2013; 31(31): 3935-3943. [PubMed: 24043745]

95. Mueller A, Bachmann E, Linnig M, et al. Selective PI3K inhibition by BKM120 and BEZ235 alone or in combination with chemotherapy in wild-type and mutated human gastrointestinal cancer cell lines. Cancer Chemother Pharmacol. 2012; 69(6):1601-1615. [PubMed: 22543857]

96. Serra V, Markman B, Scaltriti M, et al. NVP-BEZ235, a dual PI3K/mTOR inhibitor, prevents PI3K signaling and inhibits the growth of cancer cells with activating PI3K mutations. Cancer Res. 2008; 68(19):8022-8030. [PubMed: 18829560]

97. Yao F, Kausalya JP, Sia YY, et al. Recurrent Fusion Genes in Gastric Cancer: CLDN18ARHGAP26 Induces Loss of Epithelial Integrity. Cell Rep. 2015; 12(2):272-285. [PubMed: 26146084]

98. Schuler MH, Zvirbule Z, Lordick F, et al. Safety, tolerability, and efficacy of the first-in-class antibody IMAB362 targeting claudin 18.2 in patients with metastatic gastroesophageal adenocarcinomas. Paper presented at: ASCO Annual Meeting Proceedings. 2013

99. Sahin U, Koslowski M, Dhaene K, et al. Claudin-18 splice variant 2 is a pan-cancer target suitable for therapeutic antibody development. Clin Cancer Res. 2008; 14(23):7624-7634. [PubMed: 19047087]

100. Al-Batran S-E, Schuler MH, Zvirbule Z, et al. FAST: An international, multicenter, randomized, phase II trial of epirubicin, oxaliplatin, and capecitabine (EOX) with or without IMAB362, a first-in-class anti-CLDN18. 2 antibody, as first-line therapy in patients with advanced CLDN18. $2+$ gastric and gastroesophageal junction (GEJ) adenocarcinoma. Paper presented at: ASCO Annual Meeting Proceedings. 2016

101. Derks S, Liao X, Chiaravalli AM, et al. Abundant PD-L1 expression in Epstein-Barr Virusinfected gastric cancers. Oncotarget. 2016

102. Ma C, Patel K, Singhi AD, et al. Programmed Death-Ligand 1 Expression Is Common in Gastric Cancer Associated With Epstein-Barr Virus or Microsatellite Instability. Am J Surg Pathol. 2016

103. Muro K, Chung HC, Shankaran V, et al. Pembrolizumab for patients with PD-L1-positive advanced gastric cancer (KEYNOTE-012): a multicentre, open-label, phase 1b trial. Lancet Oncol. 2016

104. Le DT, Bendell JC, Calvo E, et al. Safety and activity of nivolumab monotherapy in advanced and metastatic (A/M) gastric or gastroesophageal junction cancer (GC/GEC): results from the CheckMate-032 study. Paper presented at: Proc Am Soc Clin Oncol. 2016

105. Chung HC, Arkenau H-T, Wyrwicz L, et al. Avelumab (MSB0010718C; anti-PD-L1) in patients with advanced gastric or gastroesophageal junction cancer from JAVELIN solid tumor phase Ib trial: Analysis of safety and clinical activity. Paper presented at: ASCO Annual Meeting Proceedings. 2016

106. Maron, SBLJ., Hovey, R., Bao, R., Gajewski, TF., Ji, Y., Seiwert, TY., Catenacci, DVT. Molecular Characterization of T-Cell-Inflamed Gastric Carcinoma. WIN Symposium; 2016; Paris, France.

107. Bockorny B, Pectasides E. The emerging role of immunotherapy in gastric and esophageal adenocarcinoma. Future Oncol. 2016; 12(15):1833-1846. [PubMed: 27166503]

108. Melero I, Hervas-Stubbs S, Glennie M, Pardoll DM, Chen L. Immunostimulatory monoclonal antibodies for cancer therapy. Nat Rev Cancer. 2007; 7(2):95-106. [PubMed: 17251916]

109. Ralph C, Elkord E, Burt DJ, et al. Modulation of lymphocyte regulation for cancer therapy: a phase II trial of tremelimumab in advanced gastric and esophageal adenocarcinoma. Clin Cancer Res. 2010; 16(5):1662-1672. [PubMed: 20179239]

110. Moehler MH, Cho JY, Kim YH, et al. A randomized, open-label, two-arm phase II trial comparing the efficacy of sequential ipilimumab (ipi) versus best supportive care (BSC) following first-line (1L) chemotherapy in patients with unresectable, locally advanced/metastatic (A/M) gastric or gastro-esophageal junction (G/GEJ) cancer. ASCO Meeting Abstracts. 2016; 34(15_suppl):4011. 
111. Catenacci DV. Expansion platform type II: testing a treatment strategy. Lancet Oncol. 2015; 16(13):1276-1278. [PubMed: 26342235]

112. Cunningham Dea. Phase III, randomized, double-blind, multicenter, placebo $(\mathrm{P})$-controlled trial of rilotumumab (R) plus epirubicin, cisplatin and capecitabine (ECX) as first-line therapy in patients (pts) with advanced MET-positive (pos) gastric or gastroesophageal junction (G/GEJ) cancer: RILOMET-1 study. J Clin Oncol. 2015; 33(Suppl) 


\section{Key Points}

- Anti-HER2 trastuzumab therapy is standard for HER2 amplified/ overexpressed gastroesophageal adenocarcinoma, while second/later lines of anti-HER2 directed therapy have not demonstrated definitive benefit to date.

- $\quad$ Anti-VEGFR2 ramucirumab therapy has demonstrated modest survival benefit as monotherapy and in combination with paclitaxel in second line gastroesophageal adenocarcinoma patients.

- $\quad$ Anti-EGFR therapy has not demonstrated benefit in unselected gastroesophageal patients in any line of therapy, while gene amplification/ overexpression has some evidence to support further investigation.

- $\quad$ Anti-MET therapy has not demonstrated benefit in 'over-expressing' gastroesophageal patients in any line of therapy, while gene amplification/ overexpression has some evidence to support further investigation.

- $\quad$ Other promising predictive biomarkers and targeted therapies, including FGFR2 and claudin18.2, require further investigation in larger trials to confirm therapeutic benefits for gastroesophageal adenocarcinoma patients. 


\section{로을}

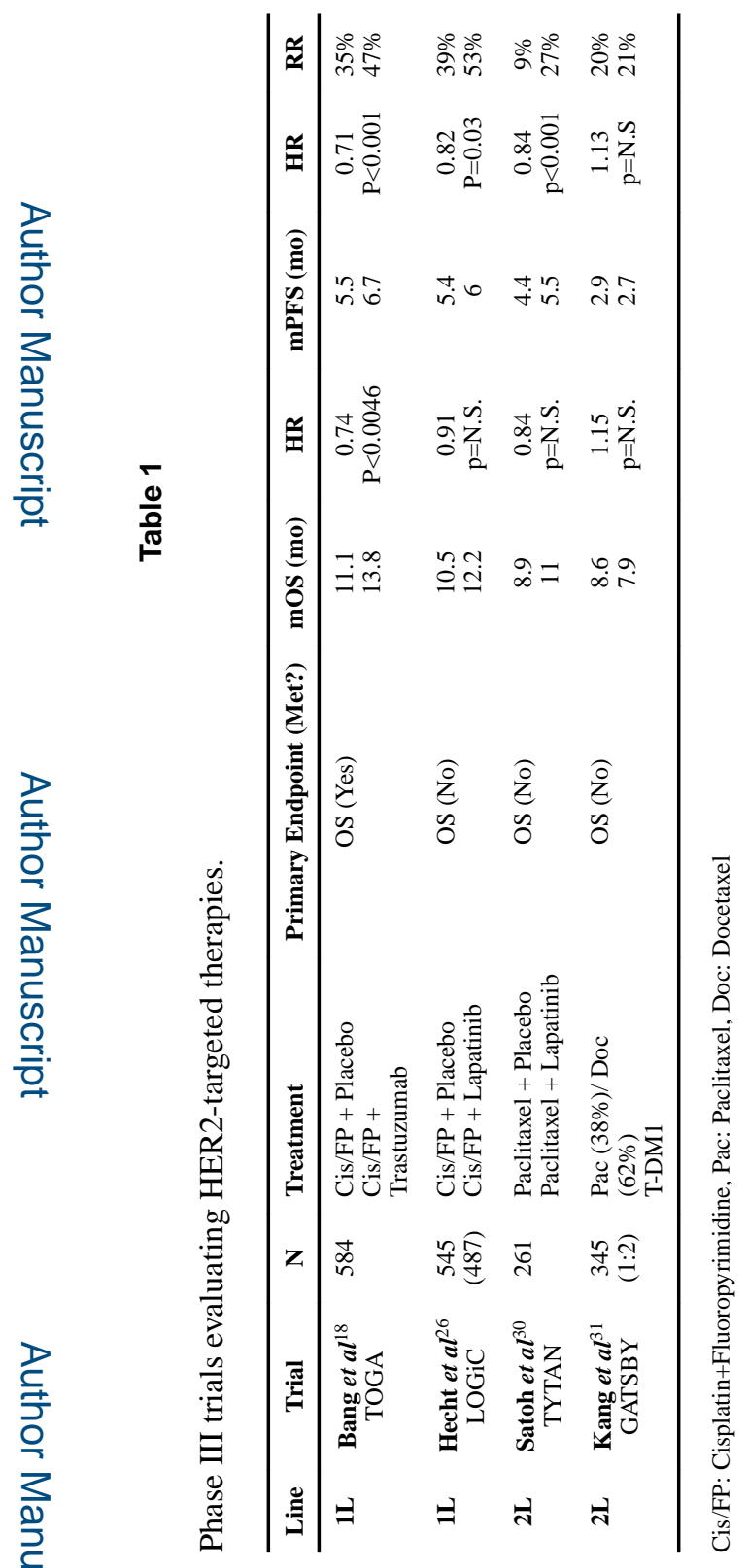




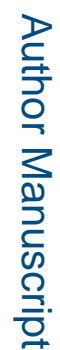

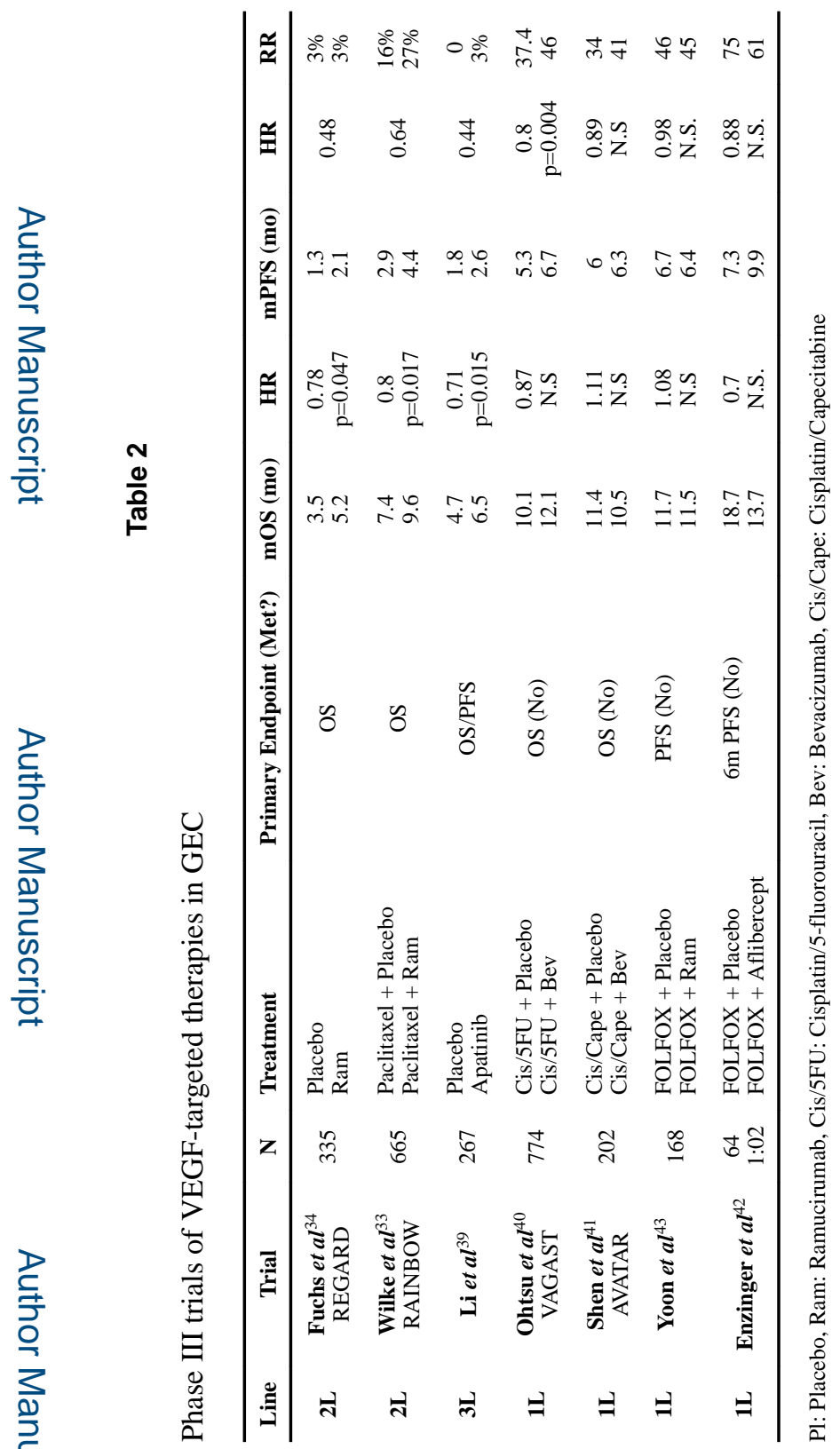

Surg Oncol Clin N Am. Author manuscript; available in PMC 2018 April 01. 


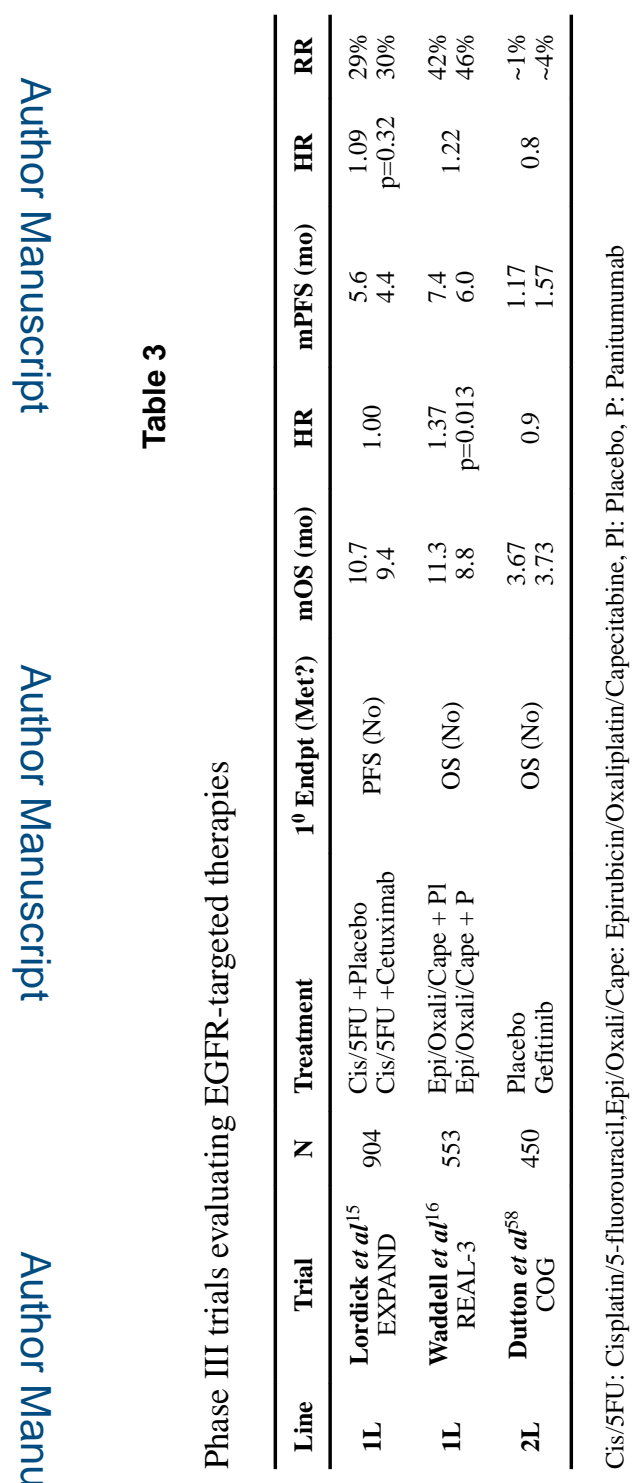

Surg Oncol Clin N Am. Author manuscript; available in PMC 2018 April 01. 


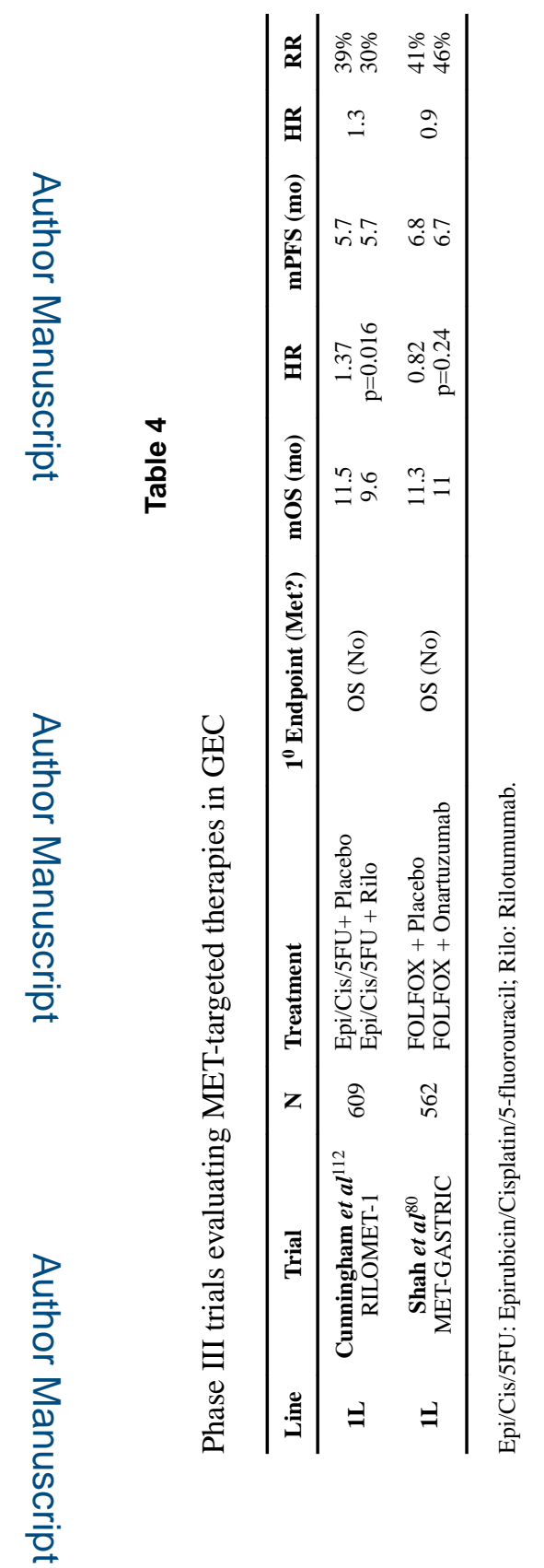

Surg Oncol Clin N Am. Author manuscript; available in PMC 2018 April 01. 


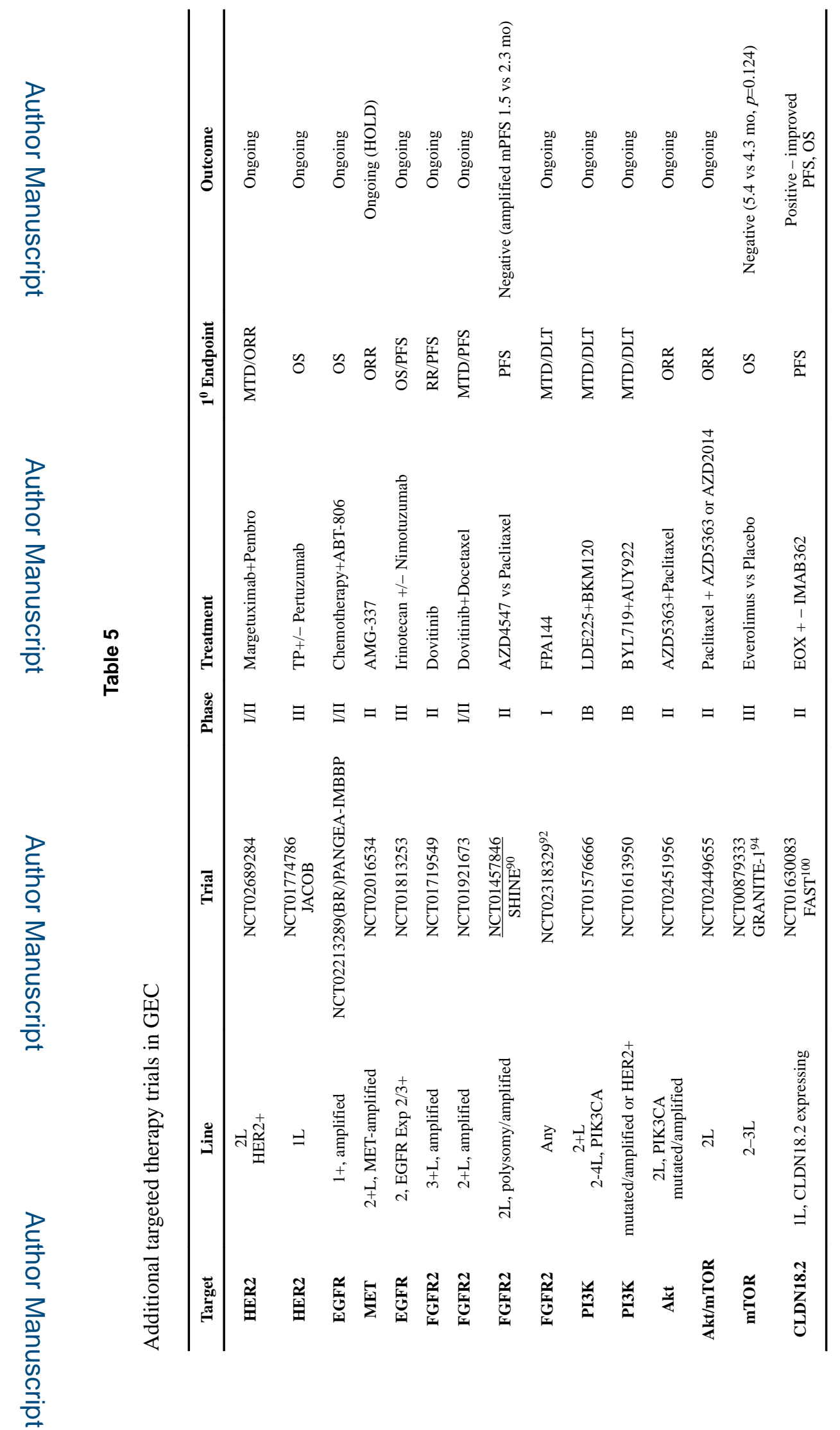

Surg Oncol Clin N Am. Author manuscript; available in PMC 2018 April 01. 